\title{
Extending rainfall-runoff models for use in environments with long-term catchment storage and forest cover changes
}

\author{
J. Hughes $^{\text {a }}$, R. Silberstein ${ }^{\text {b }}$, A. Grigg ${ }^{\text {c }}$ \\ ${ }^{\text {a } C S I R O ~ L a n d ~ a n d ~ W a t e r, ~ B l a c k ~ M o u n t a i n, ~ A C T, ~ 2601, ~ A u s t r a l i a ~}$ \\ ${ }^{\mathrm{b}}$ CSIRO Land and Water, Wembley, WA, 6913,Australia \\ ${ }^{\mathrm{c}}$ Alcoa of Australia Limited, Huntly, WA, Australia \\ Email: Justin.Hughes@csiro.au
}

\begin{abstract}
Here we present a new model algorithm based on the GR4J model (Perrin et al., 2003). The new algorithm features a threshold in the production store and a changed Evaporation calculation that allows more "memory" of catchment state from previous years. Additionally, the new algorithm accepts an input of catchment average Leaf Area Index (LAI) and can modify catchment storage and runoff with changes in forest cover.

The model was tested in catchments of the Darling Range in Western Australia. This environment has experienced both catchment drying and forest disturbance over the past 37 years. The new model algorithm shows improved predictability of run-off in test catchments as well as a better relationship between model catchment storage and observed depth to groundwater.
\end{abstract}

Keywords: Non-stationarity, groundwater, runoff, drought, long-term processes, surface watergroundwater connection, models 
J. Hughes, R. Silberstein and A. Grigg, Extending rainfall-runoff models...

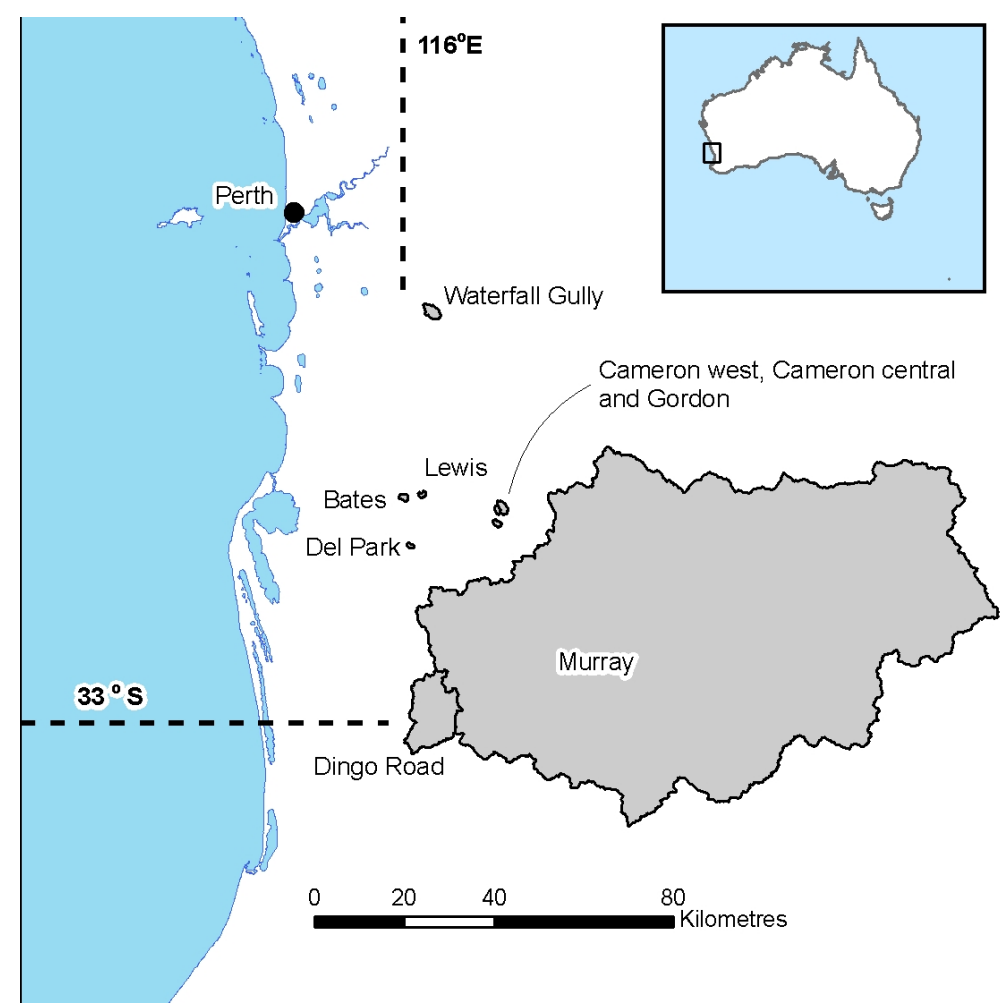

Figure 1. Study area showing Bates and Lewis catchments

\section{INTRODUCTION}

Rainfall has declined and runoff into the major reservoirs has fallen dramatically over the last 38 years in South-West Australia (Petrone et al., 2010). The continued drying of South-Western Australia have provided a test for the assumptions within all rainfall-runoff (RR) models used in the area. Runoff-ratio $(\mathrm{Q} / \mathrm{P})$ has declined during the period of drying such that, an "average" rainfall year now produces far less runoff than similar rainfall in the early seventies. This has been shown to be related to declining catchment storage, and in particular, surface-groundwater connection (Hughes et al., 2012). Typically, RR models do not have the capacity to cope with declining catchment storage and its effect on runoff. This results in over prediction of runoff in the latter portion of the observed runoff time series (and/or over prediction in the earlier part of the time series), even when calibration occurs over the range of the entire data set (CSIRO, 2009).

Building upon the hydrological research conducted at Alcoa and CSIRO in the past 5 years, this investigation attempts to advance understanding of the role of climate and hydrological processes on streamflow response in the Alcoa lease area of the Darling Range in Western Australia.

\section{METHODS}

Two catchments and accompanying data are used in this investigation. The first, Bates, is a research catchment, about $2 \mathrm{~km}^{2}$ in area. Along with streamflow and rainfall data, it has the advantage of long term piezometer data, with no change in land use across the period of monitoring. The second catchment is Lewis. Lewis is also around $2 \mathrm{~km}^{2}$ in area, with long-term groundwater, streamflow and rainfall monitoring. However, approximately $50 \%$ of Lewis was cleared for mining of bauxite in the late 1990's. This was subsequently rehabilitated with Jarrah Forest species 3 - 4 years after clearing commenced. Both catchments are located in in the Jarrah Forest of South-western Australia (Figure 1). Annual average rainfall (since 1970) is approximately $1200 \mathrm{~mm}$. However, increased frequency of drought over the last decade, in particular, has seen streamflow and groundwater levels decline (Petrone et al., 2010; Hughes et al., 2012). The regolith is around $30 \mathrm{~m}$ deep, with a surface gravelly layer of $1-10 \mathrm{~m}$ depth. 


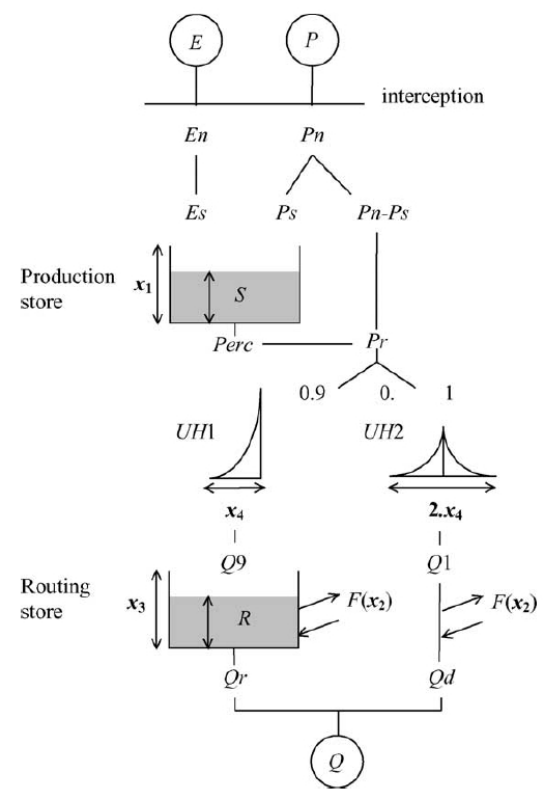

Figure 2. GR4J conceptual structure

\subsection{GR4J}

The GR4J model (Perrin et al., 2003), is widely used and relatively simple in nature. It consists of two main stores (Figure 2), the production store $(\mathrm{S})$, and the routing store $(\mathrm{R})$. Net rainfall $\left(P_{n}\right)$ and net evaporation $\left(E_{n}\right)$ are accounted for in the production store according to the current storage level on any time step according to two equations. The first calculates how much net rainfall goes into the production store

$$
P_{s}=\frac{x_{1}\left(1-\left(\frac{S}{x_{1}}\right)^{2}\right) \tanh \left(\frac{P_{n}}{x_{1}}\right)}{1+\frac{S}{x 1} \tanh \left(\frac{P_{n}}{x_{1}}\right)}
$$

where $S$ is the current level of storage ( $\mathrm{mm}), P_{n}$ is the net precipitation ( $\mathrm{mm}$ ), and $x_{1}$ is the maximum storage of $S$, and is a calibrated parameter. Such a relationship means that the input available to contribute to runoff is $P_{n}-P_{s}$.

In a similar way loss from the production store $\left(E_{s}\right)$ is calculated relative to the level of the production store $(S)$

$$
E_{S}=\frac{S\left(2-\frac{S}{x_{1}}\right) \tanh \left(\frac{E_{n}}{x_{1}}\right)}{1+\frac{S}{x 1} \tanh \left(\frac{E_{n}}{x_{1}}\right)}
$$

where $E_{n}$ is net evaporation or loss from the production store.

Changes were made to the structure of GR4J that aimed to:

1. better represent long-term reductions in runoff and storage by changing the representation of input to storage and evaporation ( Equations 1 and 2).

2. enable the model to account for changes in forest cover 
These changes were evaluated against the original GR4J code. The code changes were designed to allow the process of evaporation access to a greater amount of the production store than that affecting to $P s$ (Equation 1). In other words, implementation of a threshold below which all net rainfall would be added to the production store, while the process of evaporation, Es, continues.

To vary sensitivity of $P_{s}$ to $S$, the exponent in the numerator of Equation 1, was re-coded as a calibrated parameter of value between 1 and 2;

$$
P_{s}=\frac{x_{1}\left(1-\left(\frac{S}{x_{1}}\right)^{x_{5}}\right) \tanh \left(\frac{P_{n}}{x_{1}}\right)}{1+\frac{S}{x 1} \tanh \left(\frac{P_{n}}{x_{1}}\right)}
$$

where $x_{5}$ is the new calibrated parameter of value between 1 and 2 . Such a change can, for example, allow $P_{s}$ to be relatively reduced when $S$ approaches the value of $x_{1}$.

The updated $E s$ calculation required an additional two calibrated parameters ( $x 6$ - magnitude factor and $x 7$ shape factor), and a user defined value ( $\mathrm{mem}$ ) which determines the size of the available depletion relative to the size of the production store size available for runoff production $(x 1)$

$$
E_{s}=E_{n} * x_{6} *\left(\frac{\left(S_{\max }-x_{1}+S\right)}{S_{\max }}\right)^{x_{7}}
$$

where $S_{\max }=m e m * x_{1}$ and $m e m$ has a value greater than or equal to 1 .

This relationship allows production store levels $(S)$ to deplete to pre-defined levels, effectively increasing the influence of "catchment memory" in the simulation.

These changes were tested in Bates catchment against the original code. The model with these changes is denoted "GR4J Mod" hereafter. In Lewis catchment, where forest cover has changed over time due to mining operations, an additional change was made to the $E_{s}$ calculation (Equation 4 ) to allow it to vary with forest Leaf Area Index (LAI). The calculation of $E_{s}$ at Lewis was;

$$
E_{s}=E_{n} * x_{6} *\left(\frac{\left(S_{\max }-x_{1}+S\right)}{S_{\max }}\right)^{x_{7}} *\left(\frac{L A I_{i}}{\operatorname{mean} L A I}\right)^{x_{8}}
$$

where $x_{8}$ is a calibrated parameter, that increases or decreases the sensitivity of $E_{s}$ to LAI changes. meanLAI is a user supplied LAI value above which $E_{s}$ will increase. The model with these modifications will be denoted as "GR4J LAI" hereafter.

All models were calibrated against observed streamflow and precipitation data sourced from the West Australian Department of Water (DOW). Models were calibrated using the DREAM algorithm of Vrugt and Braak (2011), using Nash Sutcliffe Efficiency (NSE) and bias as the objective functions. LAI estimates were obtained from Alcoa of Australia Limited. Groundwater data were supplied by Alcoa of Australia Limited. Depth to groundwater was averaged by month using 41 monitoring bores in Lewis, and 9 in Bates.

\section{Results}

\subsection{Bates catchment}

Calibration of the original code and the GR4J Mod code is shown in Figure 3. NSE and bias at Bates using the original code were 0.81 , and $-0.2 \%$ respectively. This was improved with the GR4J Mod code to 0.85 and $1.1 \%$. While this would seem to be only a slight change in performance, the trend in catchment residual is greatly reduced for the modified code, implying better representation of longer term processes (Figure $3 b)$. This implies that the structure of the original code doesn't account for processes at the inter-annual scale. Significant auto-correlation in annual residual (not shown explicitly) indicates that the original model structure is inadequate at simulating processes that occur at temporal scales $>1-2$ years. The residual should consist of white noise across the frequency spectrum (all time scales). If not, the model structure may not account for processes adequately. Small differences in standard objective function measures such as NSE and bias suggest they are not a good test of model performance when longer term changes are evident.

Simulation with GR4J Mod code produced production store levels $(S)$ that were better correlated with observed groundwater levels (Figure $3 \mathrm{c}$ and $\mathrm{d}$ ). This gives the authors confidence that the structural changes in the modified code are appropriate. 

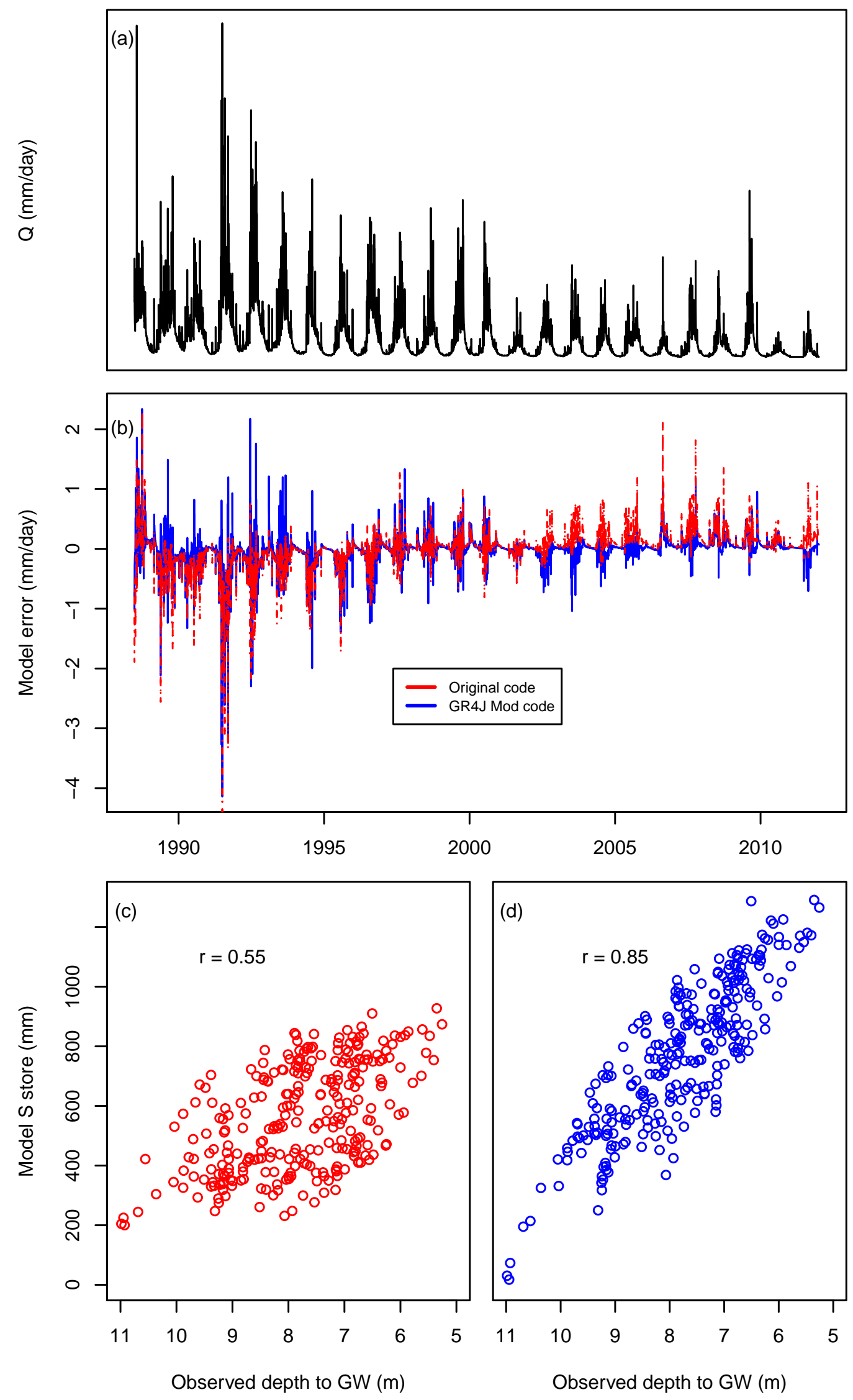

Figure 3. Comparison of the performance of GR4J with GR4J Mod at Bates catchment, (a) Observed runoff, (b) Daily model error for the original GR4J code and the GR4J Mod code, (c) Model S storage levels in relation to observed catchment average depth to groundwater for the Original code, (d) Model S storage levels in relation to observed catchment average depth to groundwater for the GR4J Mod code 

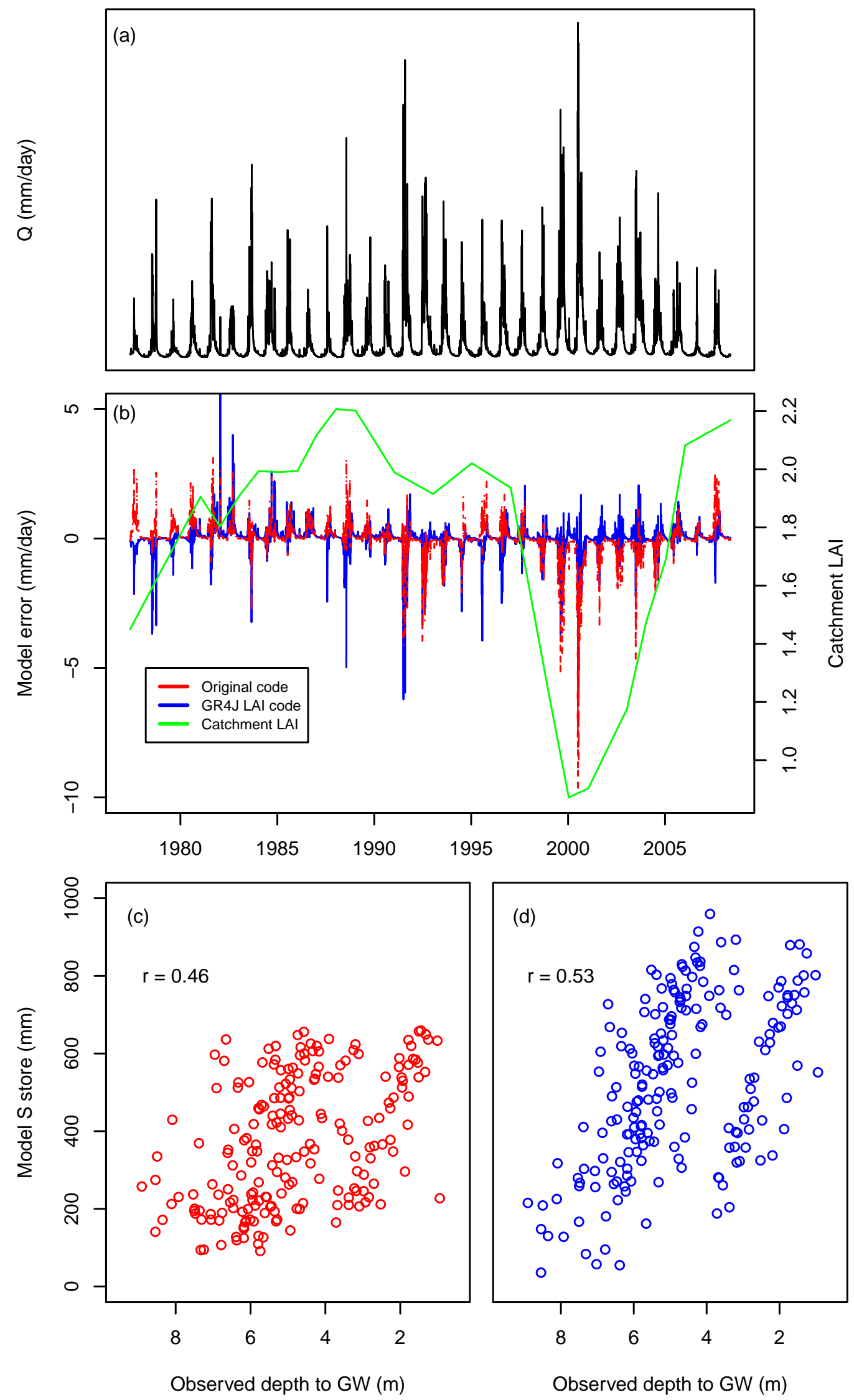

Figure 4. Comparison of the performance of GR4J with GR4J LAI at Lewis catchment, (a) Observed runoff, (b) Daily model error for the original GR4J code and the GR4J LAI code with measured catchment LAI shown, (c) Model S storage levels in relation to observed catchment average depth to groundwater for the Original code, (d)Model S storage levels in relation to observed catchment average depth to groundwater for the GR4J LAI code 


\subsection{Lewis catchment}

The addition of LAI into water balance calculations was tested at Lewis catchment with the GR4J LAI code. Obviously any code that incorporates LAI into calculation will be of benefit in catchments having experienced significant forest disturbance. NSE and bias at Lewis using the original code were surprisingly good at 0.73 and -0.2 respectively. This is since the period of major forest disturbance is only quite short relative to the full time series record. Such statistics hide structural problems such as can be seen in Figure 4b, where large errors in the original code were evident during the period of forest clearing, mining and early rehabilitation where LAI was lower. Use of the GR4J LAI code improved NSE and bias to 0.78 and $2.5 \%$ respectively. Only slight improvements were evident in the correlation between model production store $(S)$ and observed depth to GW for Lewis catchment with the updated code. This implies that either calibration and/or model structure will need to be updated to get better correlation with GW levels and runoff.

\section{CONCLUSION}

Changes made to the original GR4J algorithm show potential to better represent long-term processes in catchment hydrology. The changes require the addition of $3-4$ new parameters which, in some respects, undo the elegant simplicity of the original GR4J code. However the changes illustrate the nature of modifications required to represent longer term processes. Improved goodness of fit statistics and trends in catchment error result from modified code. Catchment storage may be better represented with modified codes as well. Further improvements are anticipated through:

1. more wide spread testing, particularly cross-validation;

2. improvement of calibration processes;

3. more appropriate objective functions during calibration

\section{ACKNOWLEDGEMENT}

This work is funded by Alcoa of Australia Limited and CSIRO Land and Water.

\section{REFERENCES}

CSIRO (2009). Surface water yields in south-west Western Australia. A report to the Australian Government from the CSIRO South-West Western Australia Sustainable Yields Project. Technical report, CSIRO Water for Healthy Country Flagship.

Hughes, J. D., K. C. Petrone, and R. P. Silberstein (2012). Drought, groundwater storage and streamflow decline in southwestern Australia. Geophysical Research Letters 39. L03408, doi: 10.1029/2011GL050797.

Perrin, C., C. Michael, and V. Andreassian (2003). Improvement of a parsimonious model for streamflow simulations. Journal of Hydrology 279, 275-289.

Petrone, K. C., J. D. Hughes, T. G. V. Neil, and R. P. Silberstein (2010). Streamflow decline in southwestern Australia, 1950 - 2008. Geophysical Research Letters 37. L11401, doi: 10.1029/2010GL043102.

Vrugt, J. A. and C. J. F. T. Braak (2011). DREAM(D): an adaptive Markov Chain Monte Carlo simulation algorithm to solve discrete, noncontinuous, and combinatorial posterior parameter estimation problems. Hydrology and Earth System Science 15, 3701-3713. doi:10.5194/hess-15-3701-2011. 